\title{
Supporting Information 3: Simulations to aid in the design of microbes for synthesis of metallic nanomaterials
}

\author{
Kyle L. Naughton ${ }^{1}$, James Q. Boedicker ${ }^{1,2}$ \\ ${ }^{1}$ Department of Physics and Astronomy, University of Southern California, Los Angeles, CA, \\ USA. \\ ${ }^{2}$ Department of Biological Sciences, University of Southern California, Los Angeles, CA, USA. \\ Example Code for Figure 2 in the Main Text: As(V) Reduction
}

$\circ==============$ Simulations to aid in the design of microbes for
synthesis of metallic nanomaterials $==============\circ$
$\%$ Naughton and Boedicker 2021
$\%==============$ ABOUT THIS CODE \& THEORY $==============\%$
$\frac{\circ}{\text { Classical Nucleation Theory (CNT) dictates that NP formation can be broken up }}$ into 3 parts:

(i) chemical reaction

(ii) nucleation

(iii) growth

The whole process is driven by the supersaturation "S" (oversaturation) of atomic monomers that compose the material.

The Gibbs free energy, which directs nucleation, depends primarily on the interfacial free energy "gamma"

For a detailed explanation of theory see Thanh, Maclean, \& Mahiddine Chem Rev 2014, https://pubs.acs.org/doi/10.1021/cr400544s

This code appends biological perturbation to CNT

- Compartmentalization into three regions (extracellular, periplasm, cytoplasm)

- Transport between those three regions, where transport is dicated by Michaelis-Menton (MM) kinetics

https://en.wikipedia.org/wiki/Michaelis\%E2\%80\%93Menten kinetics

- Reduction of pre-cursor materials (e.g. of Pd(II) -> Pd(0)), which is described by a 1 st order $\operatorname{rxn}(\mathrm{d}[\mathrm{X}(0)] / \mathrm{dt}=+\mathrm{n}$ reductases*k reduction*[X(II)]

- Nucleation, which is driven by a reduction in the interfacial free energy

"gamma" and the availability of nucleation sites "nuc_sites" in each region. The variable "theta" accounts for the potential reduction of the interfacial energy via heterogeneous nucleation on biomolecules.

One of the interesting perturbations added is that homogenous, spontaneous nucleation of NP competes with heterogenous nucleation, which occurs on defect or, importantly, a metal binding peptide (e.g. CDS7 from Peelle et al Acta Biomater 2005)

Another key ingredient is the transport across the membranes. The rate is given by "Vmax" and the rate can be controlled for each membrane for each chemical species. That means that Zn2+ travels differently than S2- and that both species travel differently across each membrane. 
See the variable "Vmax", which has two columns: 1 for extracell-periplasm

$(\mathrm{OM})$ barrier and 2 for the periplasm-cytoplasm (IM) barrier

운

$\div===============$ DETAILS ABOUT THIS CODE $===============0$

$\frac{\circ}{0}\{$

* most units are moles/L, meters, seconds, and grams

VARIABLES

$\mathrm{h}=$ size of time step (in seconds), "h" is from Euler forward literature Nsteps = number of hours of the simulation

$i=$ number of steps

$l$ = region (extra, peri, cyto)

$\mathrm{k}=$ homogenous or heterogenous nucleation $(1,2)$

$\mathrm{y}(\mathrm{i}, \mathrm{s}, 1, \mathrm{k})=$ molar concentration of species $1,2,3,4,5$

Nanoparticle Radius $(i, s, 1, k)$ = radius, each column is a time step containing all radii

Nanoparticle Number $(i, s, 1, k)=$ number, each column is a time step containing number of all radii

The code used the Euler forward method and can divided into 4 steps

Step 0: Time, Concentrations, Parameters defined

Step 1: The concentration of each species is updated, transport and chemical reduction occur

Step 2: Nucleation occurs. Homogenous and heterogenous (heterogenous

nucleation requires unoccupied nucleation sites > 0) occur

Step 3: Nanoparticle growth occurs.

The code outputs a big array of the number of NPs "Number" in each region "l" and the radius of NPs "Radius"

The data can be cleaned up to erase NP that are smaller than an atom or when there is less than 1 of something

The data are binned into nbins in an array called "ed" for each region. A histogram can be displayed (figure 696, figure 601)

A Gaussian Fit is attempted to the binned data The concentration of each species can be displayed (Figure 3)

The nucleation rate (dN) and total number of nuclei can be displayed (Figure $76)$

The code prints the key parameters used in the terminal and lets you know what you did or did not turn of

TURNING ON Or OFF PERTUBATIONS

Nucleation, transport, and single-metal reduction can be turned on or off using logical Matlab structures

Example: "transport.true = 1" turns on transport, otherwise all the parameters are set to 0 
Example: "metal reduction.true = 1" turns of reduction of a single metal species, like $\mathrm{P} \overline{\mathrm{d}}(\mathrm{II})$

Example: "hetero nucleation.true $=1$ " turns on nucleating sites, which you define by changing "theta" and "nuc sites"

* For nucleation you can set an upper limit on the number of sites available with "nucleation.enforce number" (assume peptides keep NP attached) or allow for NP to detach, which effectively means there is no limit on the number of nucleation

sites

CORE PARAMETERS

"gamma" (interfacial energy)

"kl" (the chemical reaction rate)

"C0_sat" (the solubility of the material)

"kgr" (the basal growth rate constant of the material)

"Vmax" (the transport rate of the materials, when transport on)

the reduction rate of $\mathrm{X}$ to $\mathrm{X}^{\prime}$ with "nr X" or "kr X" (number and rate)

\section{CONCENTRATION}

Enter the concentration in millimoles of your chemicals. code automatically converts "1.0" to "0.001" moles/L

$1=2$

$1=3$

$l=1$

y(time step, type of chemical, compartment) = [initial conc in extracellular space, initical Conc in periplasm, initial conc in cytoplasm]

\section{TRANSPORT}

Because the system has different volumes and concentrations, Michaelis-Menton (MM) kinetics works best.

Eqn (N trans + leaky $) * \operatorname{Vmax}^{*}(\mathrm{y} 2-\mathrm{y} 1) /(\operatorname{Vol}(11) *(\mathrm{Km}+\mathrm{y} 1+\mathrm{y} 2))$

The paramters are $\operatorname{Vmax}$ (max rate), Km (conc of substrate at half-max rate),

$\mathrm{N}$ trans, the relative number of transporters $=0->1$

léaky, leaky transport rate $=0->1$, which can be turned off

NUCLEATION

A key parameter is gamma. In the presence of unoccupied nucleation sites, gamma is reduced for heterogeneous nucleation by a factor theta

\section{REDUCTION}

Pre-precursors are $y(:, 4,:)$ and $y(:, 5,:)$, which can be reduced to y $(:, 1,:)$ and $\mathrm{y}(:, 2,:)$ respectively y4 -> yl: driven by $\operatorname{Vmax}(6), \mathrm{Km}(6)$, placed in the periplasm by writing dy/dt $=\ldots+\operatorname{Vmax}(6){ }^{*} y(i, 4,2) /(\operatorname{Km}(6)+y(i, 4,2))$ where "y(i,4,2)" refers to the i-th time steps, species 4 (e.g. As (V)) and region 2, periplasm 


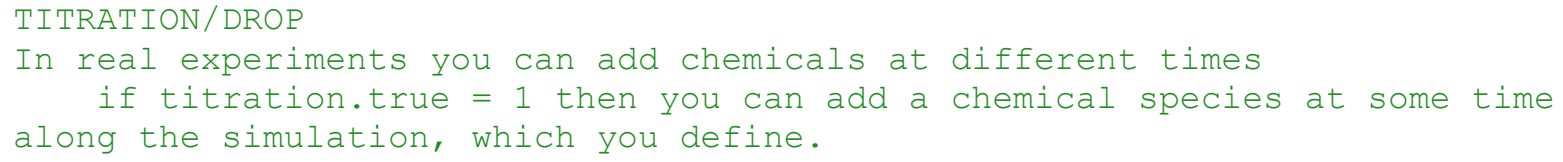

- Expression of 10^4 nucleating peptides in the periplasm that reduce the barrier to nulceation

- Expression of periplasmic, innermembrane-bound As(V) reductase that converts As (V) to As (III)

Expected outputs are 3 figures with the Radius probability distribution, number of nanoparticles, and concentrations of reactants

응

ㅇ---- Start of Code -----

close all closes all figures

응------- DEFINE TIME STEPS --------

$\mathrm{h}=10 ; \%$ time step in seconds

Nsteps $=16$; $\frac{\circ}{\circ}$ number of hours of simulated time

Nsteps $=$ Nsteps $\star 3600 / \mathrm{h} ; \%$ convert to steps

time $=(1:$ Nsteps $) * h / 3600 ;$ o time array in hours units for plotting $\mathrm{u}=$ 'Time [hr]'; $\%$ for labeling plots when they are output formatSpec $=' \frac{\circ}{\circ} .1 e^{\prime} ;$ o number of signifigant figures to display in Command Window 





o parameter from Talapin et al 2002 that appears in dR/dt growth equation alpha $=0.5$; $\frac{\circ}{0}$ for growth rate from Talapin et al 2002

o Basal growth rate of material. NOTE: You can change for homo and heterogenous nucleation+growth

$\operatorname{kgr}=[1 e-5 ; 1 e-5] ;$ o growth rate for homogenous and heterogenous nucleation $\mathrm{m} / \mathrm{s}$, value similar to that used in Chen et al 2015

\% Interfacial energy of CO

gamma $=0.125$; $\frac{\circ}{\circ}$ used in Talapin et al 2002

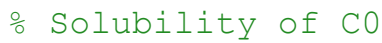

c0_sat $=5 e-8$; $\frac{\circ}{0}$ Saturation conc./ solubility of ZnS

$\left.\frac{\circ}{0}\right\}$

ㅇ------------- PRECURSOR PARAMETERS in milli-moles/L -------------

$\mathrm{y}(1,1,:)=[0.0 ; 0.0 ; 0.0] * 1 e-3 ;$ moles of precursor $y 1, e .9$. Zn $2+$,

$\mathrm{y}(1,2,:)=[1.0 ; 0.0 ; 0.0] * 1 e-3 ;$ o moles of precursor y2, e.g. S2-

$y(1,3,:)=[0.0 ; 0.0 ; 0.0] * 1 e-3 ;$ moles of monomer, e.g. ZnS0

$\mathrm{y}(1,4,:)=[1.0 ; 0.0 ; 0.0] * 1 e-3 ; \%$ moles of pre-precursor

$\mathrm{y}(1,5,:)=[0.0 ; 0.0 ; 0.0] * 1 e-3 ;$ \% moles of pre-precursor

응 ------------- TRANSPORT PARAMETERS -------------

transport.true $=1 ;$ make $==1$ to allow metal ion transport

transport.leaky $=0 ;$ make $=1$ to allow leaky transport in the absence of $\mathrm{N}$ trans, the number of transport proteins.

if transport.true $==1$

\% relative number of transporters per membrane (0 to 1 for min to max)

o column 1 is outer membrane, column 2 is inner memrane

$\mathrm{N}$ trans $=\left[\begin{array}{ll}1 & 0.2 ;\end{array}\right.$ \% for Species 1

$10.8 ;$ 응 species 2

0 ; ; for species 3

10.2 ; $\%$ for Species 4

$10.8]$; $\frac{\circ}{0}$ for Species 5

\% Example: $N$ trans $(5,1)$ is the number/max for Species 5 bt extracell and periplasm on outer membrane

else

N_trans $=$ zeros $(5,2) ;$ o transport off (leaky still possible)

end

o Assume Michaelis-Menton Kinetics, which uses Vmax (max rate) and Km (conc. of half-maximum rate of substrate/ precursor)

$\operatorname{Vmax}=[1.995 e-23 ;$ ion transport rate $\mathrm{Y} 1, \mathrm{e} . \mathrm{g}$. Zn2+ or Cd2+

$8 e-23$; $\%$ ion transport rate $Y 2$, e.g. S2-

0 ; $\frac{\circ}{0}$ ion transport $Y 3$, the insoluble metal species

$2 e-23$; $\frac{\circ}{\circ}$ ion transport pre-precursor of Y4, e.g. As (V)

6e-22]; $\frac{\circ}{0}$ ion transport pre-precursor of Y5, e.g. thiosulfate

$\mathrm{Km}=[0.71 \mathrm{e}-6 ; \% \mathrm{~mole} / \mathrm{L}$

$1 e-6 ; \% \mathrm{~S} 2-$

0 ; insoluble monomers of, for example, ZnS[0] or Pd[0]

$0.71 e-6 ; \%$ e.g. As (V)

$0.71 e-6] ; \%$ e.g. thiosulfate 
LEAKY TRANSPORT PARAMETERS ---------------

\% Note: In general, transport can be leaky despite no transport proteins being expressed. This gives the user the ability to allow leaky transport o in addition to allowing transport proteins to be expressed.

if transport.leaky $==1$

leaky $=\left[0\right.$ 0; $\frac{\circ}{0}$ leaky transport for species 1

0 ; $\%$ leaky transport for species 2

0 0; $\frac{\circ}{0}$ leaky transport for species 3

0 ; $\frac{\circ}{0}$ leaky transport for species 4

0 0 ] ; $\frac{\circ}{\circ}$ leaky transport for Species 5

\% Example: leaky $(1,2)$ is the leaky transport rate (as o of maximum rate) for Species 1 into cytoplasm from periplasm acoss inner membrane

else

leaky $=\operatorname{zeros}(5,2) ;$

end

은 -------------- METAL REDUCTION PARAMETERS ---------------

\% THIOSULFATE REDUCTION PARAMETERS --------------

nred_S $=[0 ; \%$ empty

1]; \% relative expression of reducatse for control of thiosulfate reduction (default $=1$ )

kred_S $=[0 ; \%$ empty

1e-2]; ate of thiosulfate reduction

ㅇ ARENSITE REDUCTION PARAMETERS -------------

\% number of reducatses, set 0 to 1 where 1 is maximum and $>1$ is over

expression

nred_As $=[0 ; \quad$ As $(V)$ reductase in extracellular space

$1 ; \quad \frac{\circ}{\circ}$ As $(V)$ reductase in periplasm

$0] ;$ As $(V)$ reductase in cytoplasm

kred As $=[0 ; \%$ reduction outside

$25 e-5 ;$; reduction periplasmic

$0] ; \circ$ reduction cytoplasmic

\% PALLADIUM REDUCTION PARAMETERS

metal_reduction.true $=0$;

o automatically turn off if youre not dealing $\mathrm{w} P \mathrm{Pd}$

if type $\sim=3$

end

metal reduction. true $=0$;

if metal reduction.true $==1$

$\mathrm{kred}^{-}=[5 e-5 ;$ o reduction outside

$5 e-5 ;$ \% reduction periplasmic

nred $=[1$;

$0] ; \circ$ reduction cytoplasmic

0 ;

$0]$;

end

은 -------------- NUCLEATION PARAMETERS --------------

hetero_nucleation.true = 1; 


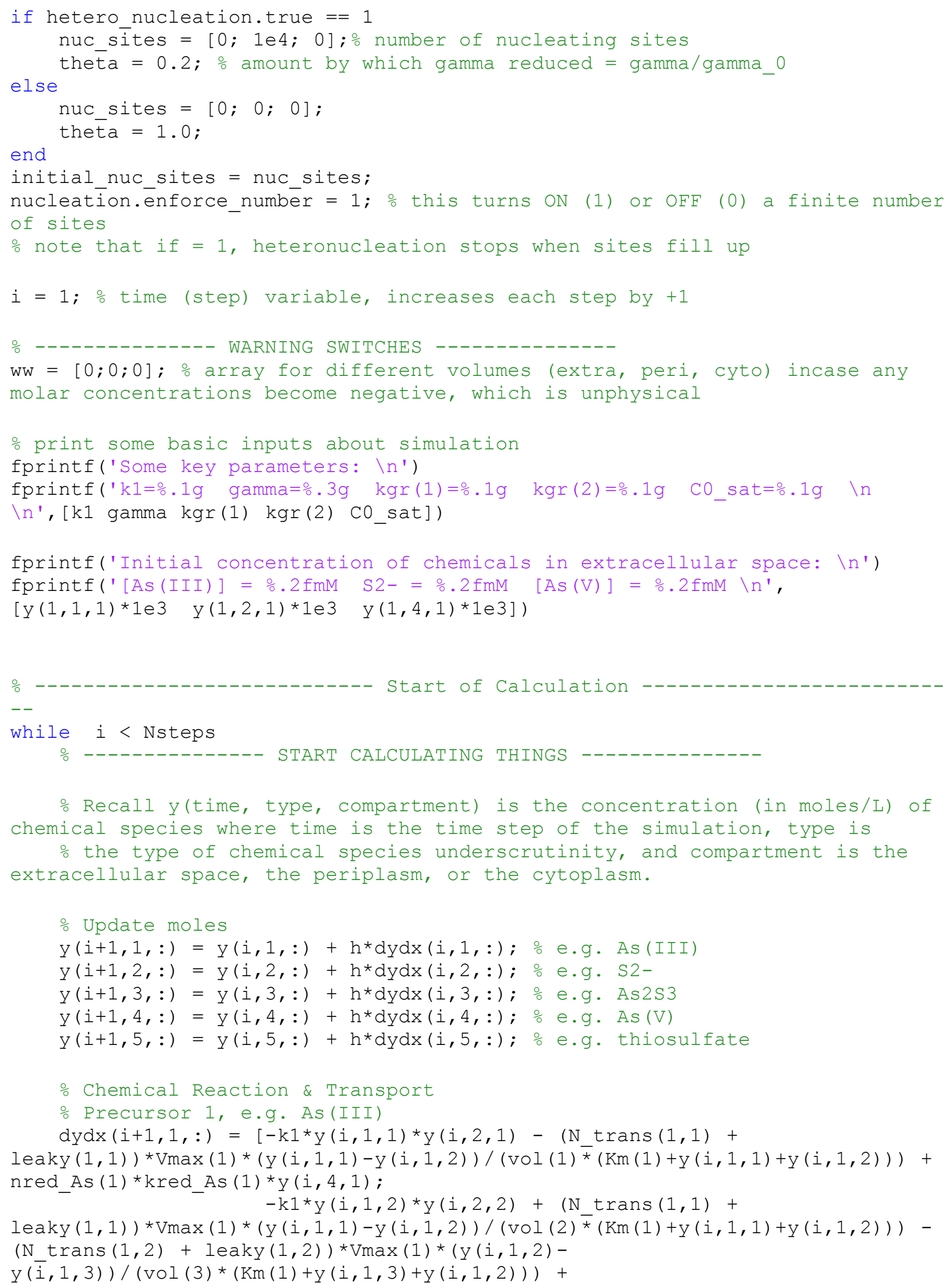




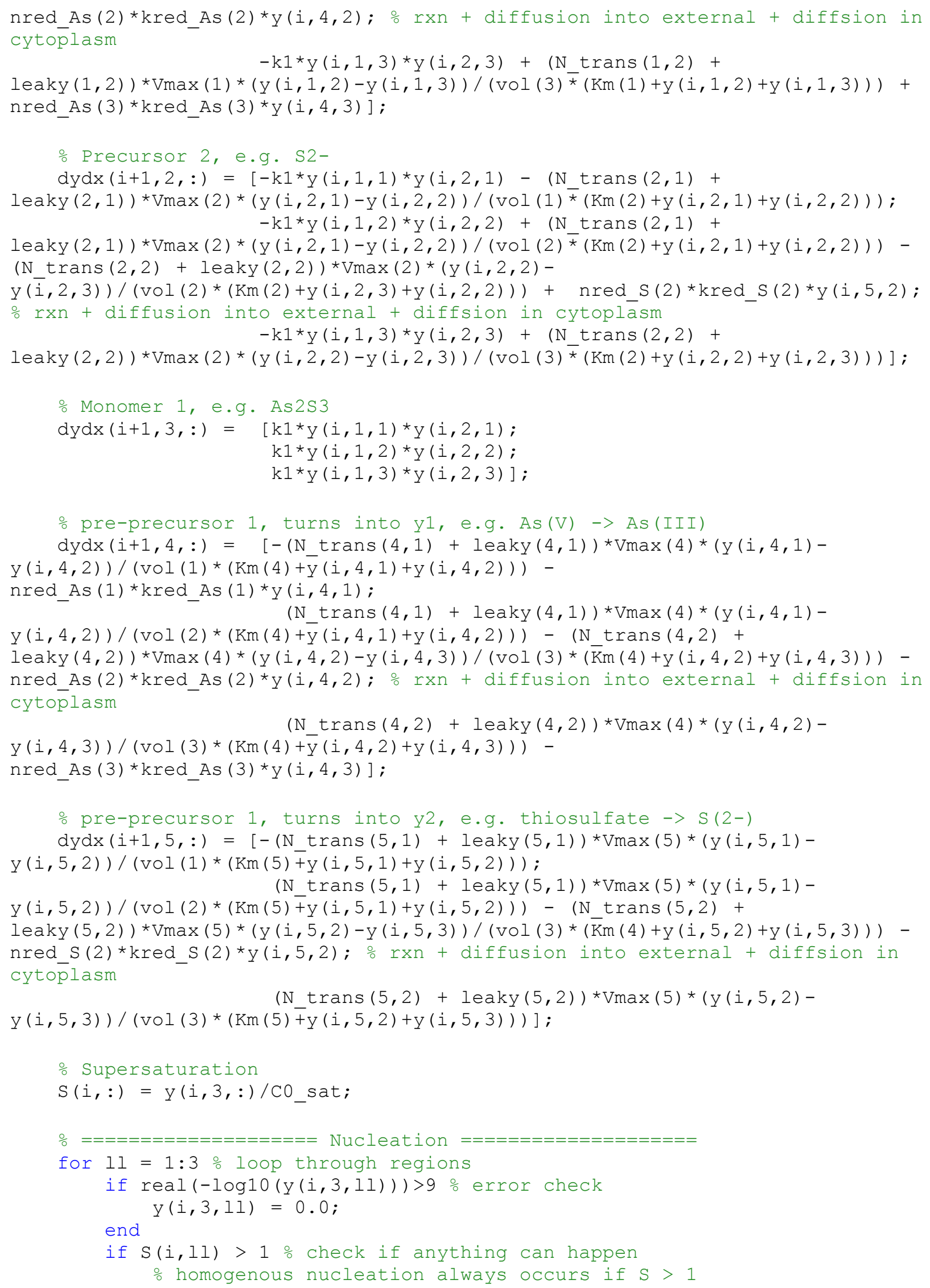




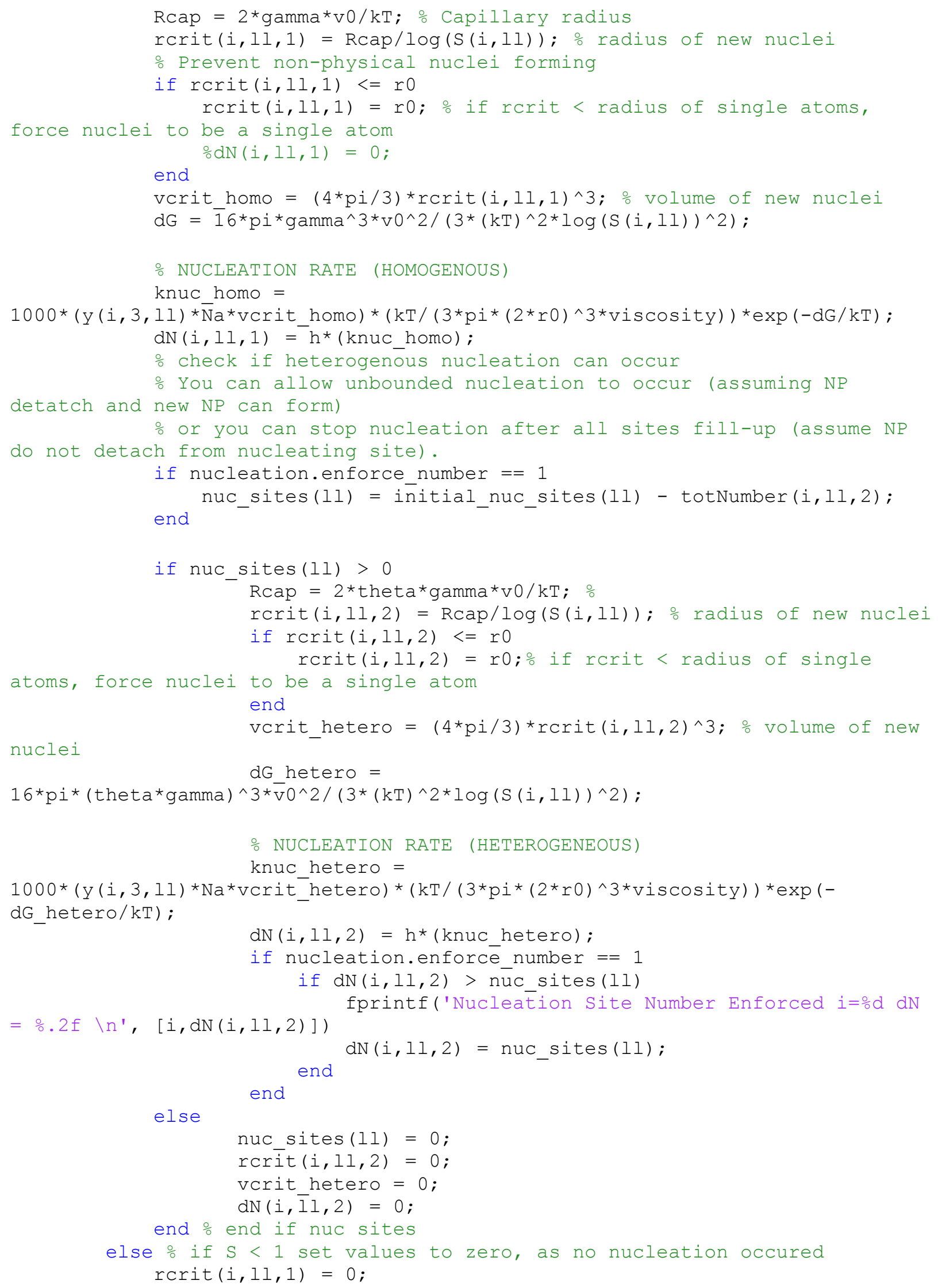




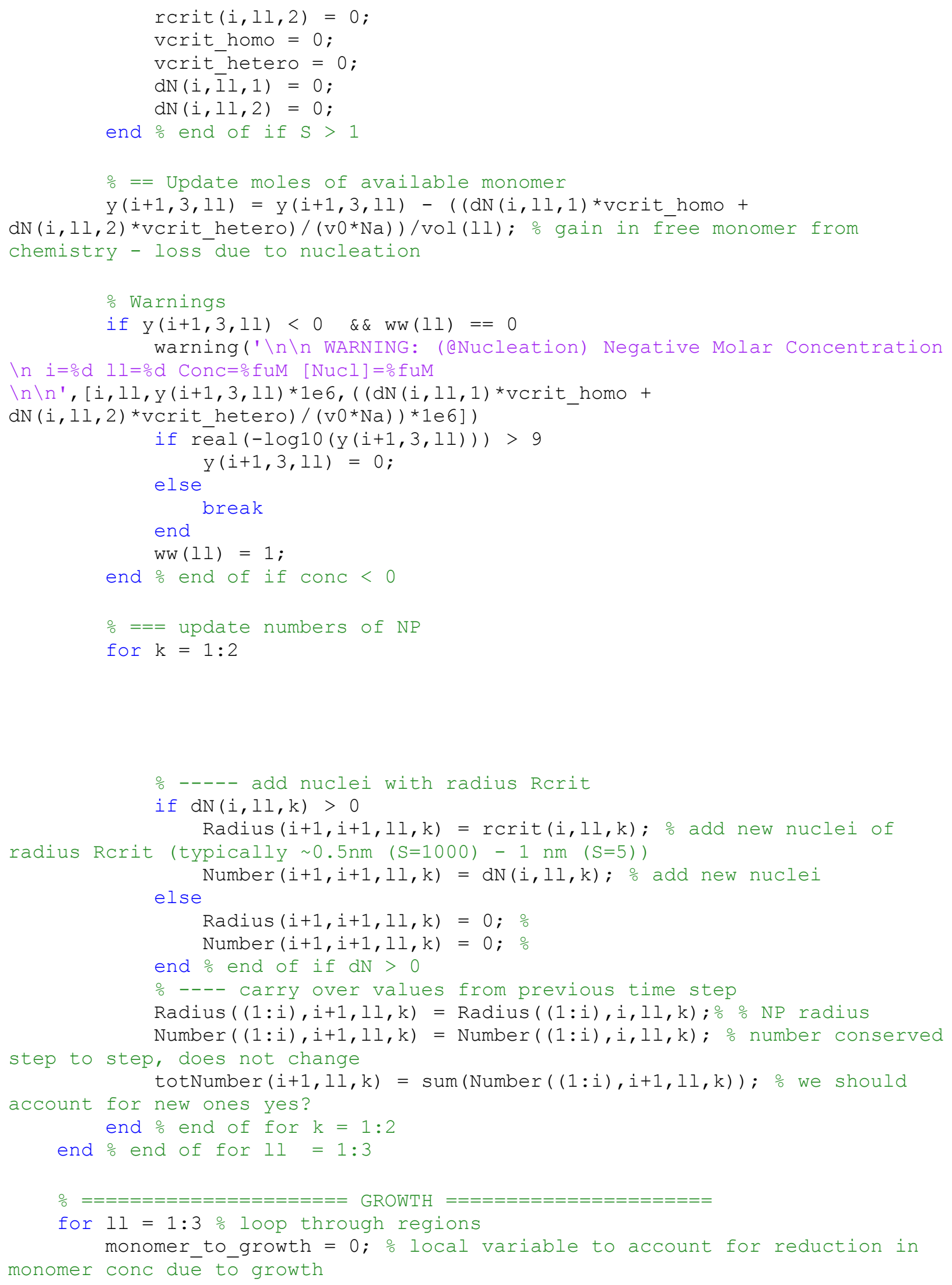




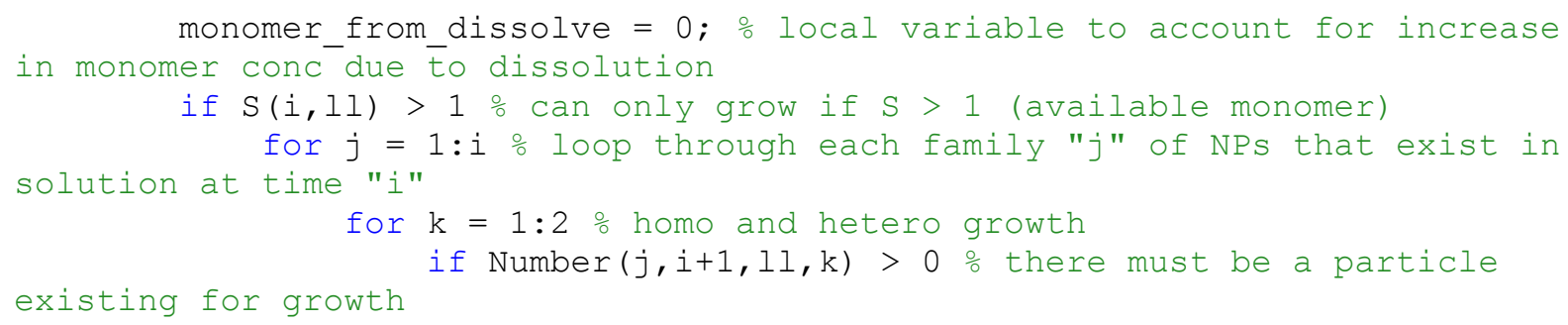




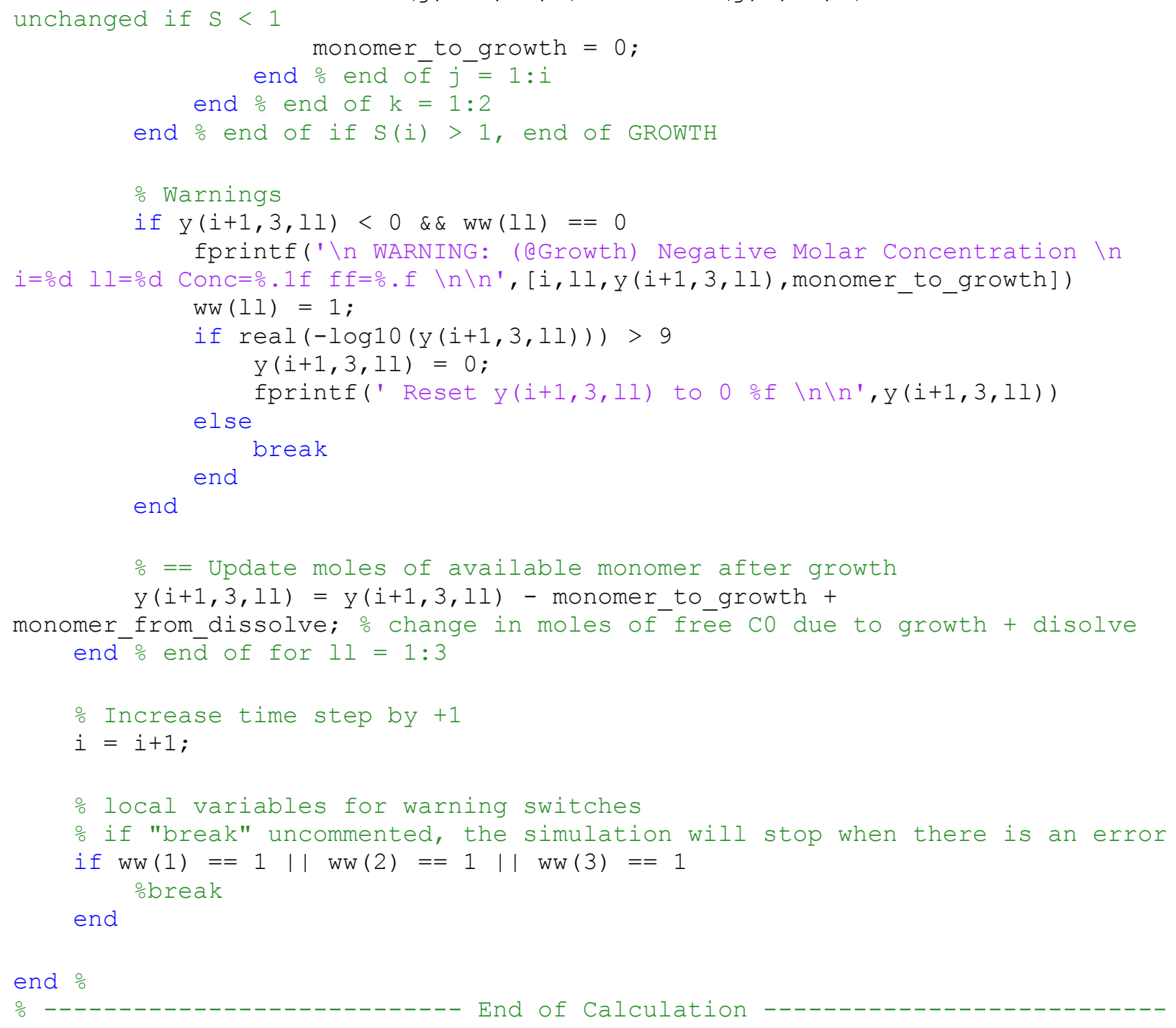


ed $(:, 1, k)=1$ inspace $(1.0$, round $(1.5 * \max (\max (\max (\operatorname{Radius}(:$, end-

$2,1, k))$ ))*(e9), nbins); 응 define the bin edges to add histograms

for $i=1$ :nbins -1

\% homo and hetero indipendent normalized

$\operatorname{PDF}(i, 1, k)=\operatorname{sum}($ Number $($ find $(\operatorname{ed}(i, 1, k)<\operatorname{Radius}(:, \operatorname{end}-2,1, k) * 1 e 9$

\& $\operatorname{Radius}(:$, end $-2,1, k) * 1 e 9<\operatorname{ed}(i+1,1, k))$, end $-2,1, k)) /$

sum (Number (find (ed $(1,1, k)<\operatorname{Radius}(:$, end-2, $1, \mathrm{k}) * 1 e 9$ \& $\operatorname{Radius}(:$, end $-2,1, \mathrm{k}) \star 1 e 9$

$<\operatorname{ed}(e n d, 1, k))$, end-2, l, k) );

end

$\operatorname{PDF}(i \operatorname{sinf}(\operatorname{PDF}(:, 1, k)), l, k)=0 ;$ o set NaNs equal to zero

o ignore nuclei when Radius < lower_cutoff

radius lower_cutoff $=1 e-9$; $\frac{\circ}{\circ}$ smallest $\mathrm{NP}$ considered

totNumber $(i, \bar{l}, k)=$

sum (Number (Radius ( $:$, i, l, k) >radius_lower_cutoff, i, l, k)) ;

end

end

\% calculate the value of and index of the maximum probability

for $1=\left[\begin{array}{lll}1 & 2 & 3\end{array}\right]$

for $k=\left[\begin{array}{ll}1 & 2\end{array}\right]$

$[\mathrm{m} \cdot \operatorname{MaxpdF}(1, \mathrm{k}), \mathrm{m} \cdot \operatorname{Max} \operatorname{Index}(1, \mathrm{k})]=\max (\operatorname{PDF}(:, 1, \mathrm{k}))$;

end

end

\% change bin edges to bin centers

for $1=\left[\begin{array}{lll}1 & 2 & 3\end{array}\right]$

for $k=\left[\begin{array}{ll}1 & 2\end{array}\right]$

for $i=1:$ length (ed)

var in gausFcn $\mathrm{X}(\mathrm{i}, 1, \mathrm{k})=\operatorname{ed}(\mathrm{i}, 1, \mathrm{k})+(\operatorname{ed}(2,1, \mathrm{k}) \operatorname{-ed}(1,1, \mathrm{k})) / 2$; 응 must match $\mathrm{x}$ end

end

\% radius distribution

figure (1)

set(gcf,'Position', [500, 10, 700, 900]);

$j j=1$;

$\mathrm{VV}=$ \{'Extracellular Homo', 'Extracelluar Hetero', 'Periplasm Homo', 'Periplasm

Hetero', 'Cytoplasm Homo', 'Cytoplasm Hetero'\};

for $1=\left[\begin{array}{lll}1 & 2 & 3\end{array}\right]$

$\mathrm{G}=\mathrm{a}(\mathrm{a} 1, \mathrm{~b} 1, \mathrm{c} 1, \mathrm{x}) \mathrm{a} 1 * \exp (-((\mathrm{x}-\mathrm{b} 1) / \mathrm{c} 1) . \wedge 2) ;$

for $k=\left[\begin{array}{ll}1 & 2\end{array}\right]$

$\mathrm{xData}=\mathrm{x}(:, 1, \mathrm{k})$;

$\mathrm{yData}=\operatorname{PDF}(:, \mathrm{l}, \mathrm{k})$;

if totNumber (end-2, l, k) > 1

ㅇ set up fittype and options.

ft = fittype ('gaussl');

opts = fitoptions ( 'Method', 'NonlinearLeastSquares' );

opts.Display = 'Off';

opts.Lower $=[0$ ed $(1,1, \mathrm{k}) 0]$;

opts. Upper $=[1 \max (\max (\max (e d))) \max (\max (\max (e d)))]$;

ed $(\mathrm{m} . \operatorname{MaxIndex}(1, \mathrm{k}), 1, \mathrm{k}) / 5]$;

opts.StartPoint $=[\operatorname{m} \cdot \operatorname{MaxPDF}(1, k) \operatorname{ed}(\max \operatorname{Mandex}(1, k), 1, k)$

[fitresult, gof] = fit( xData, yData, ft, opts ); 


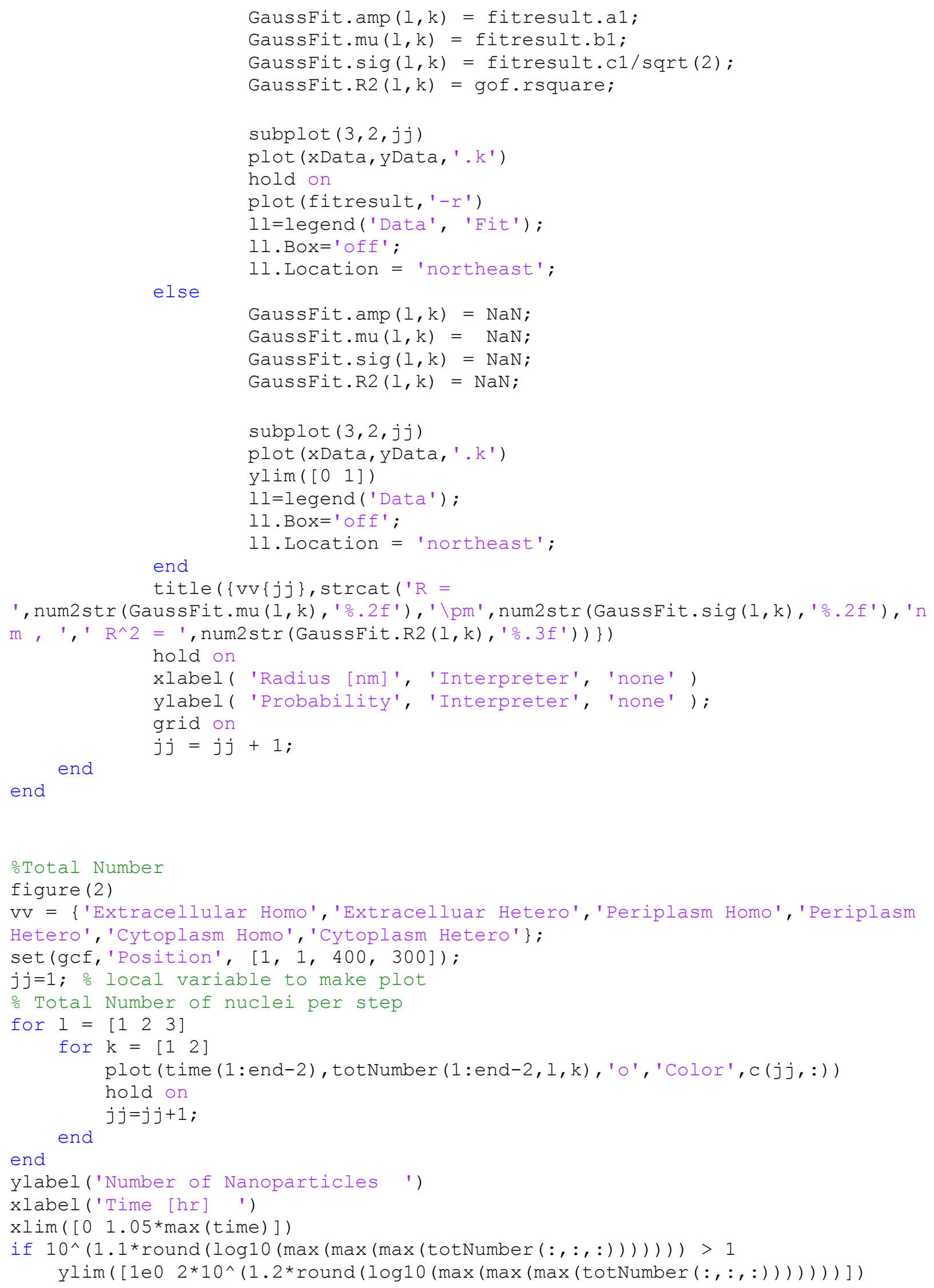




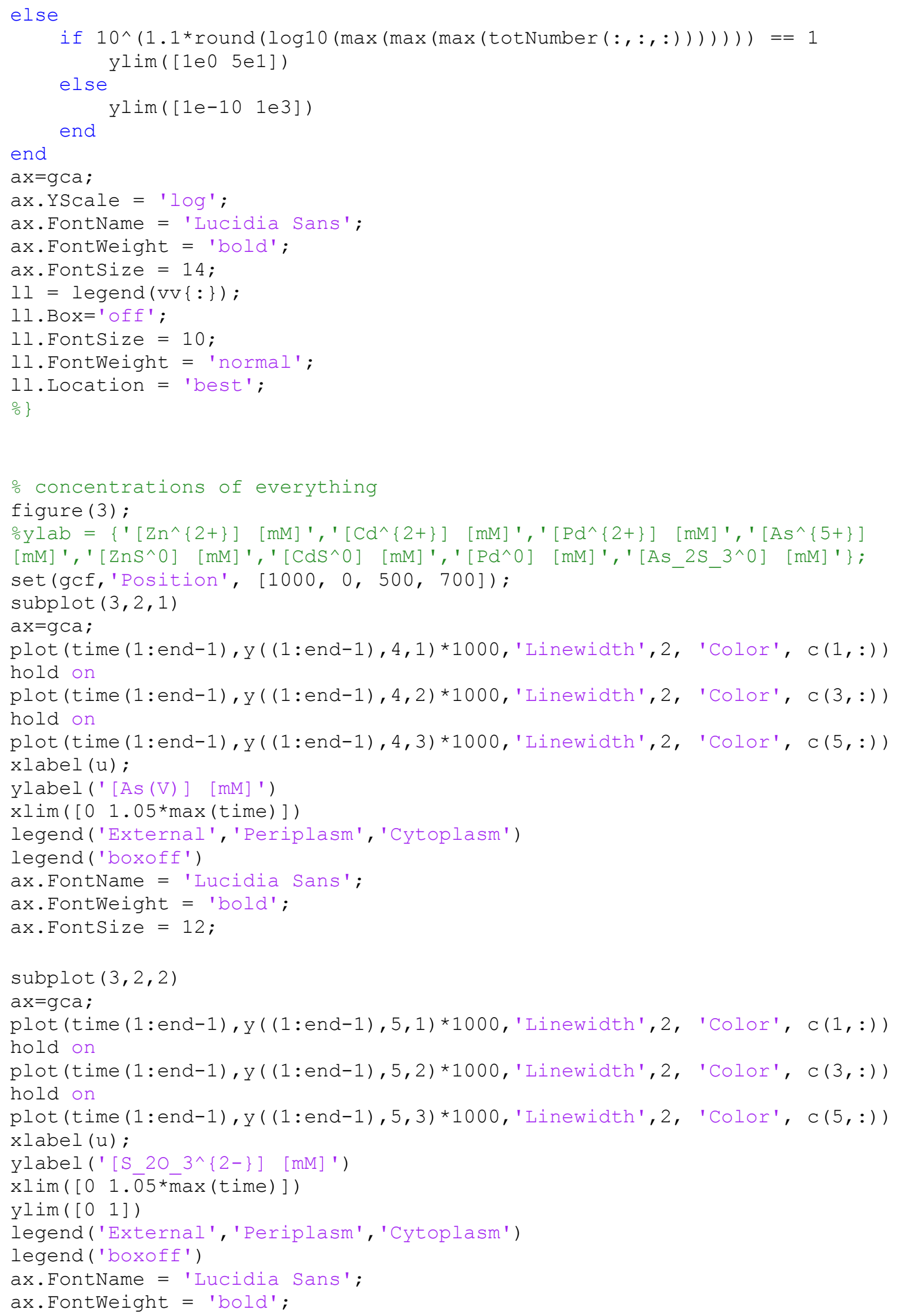




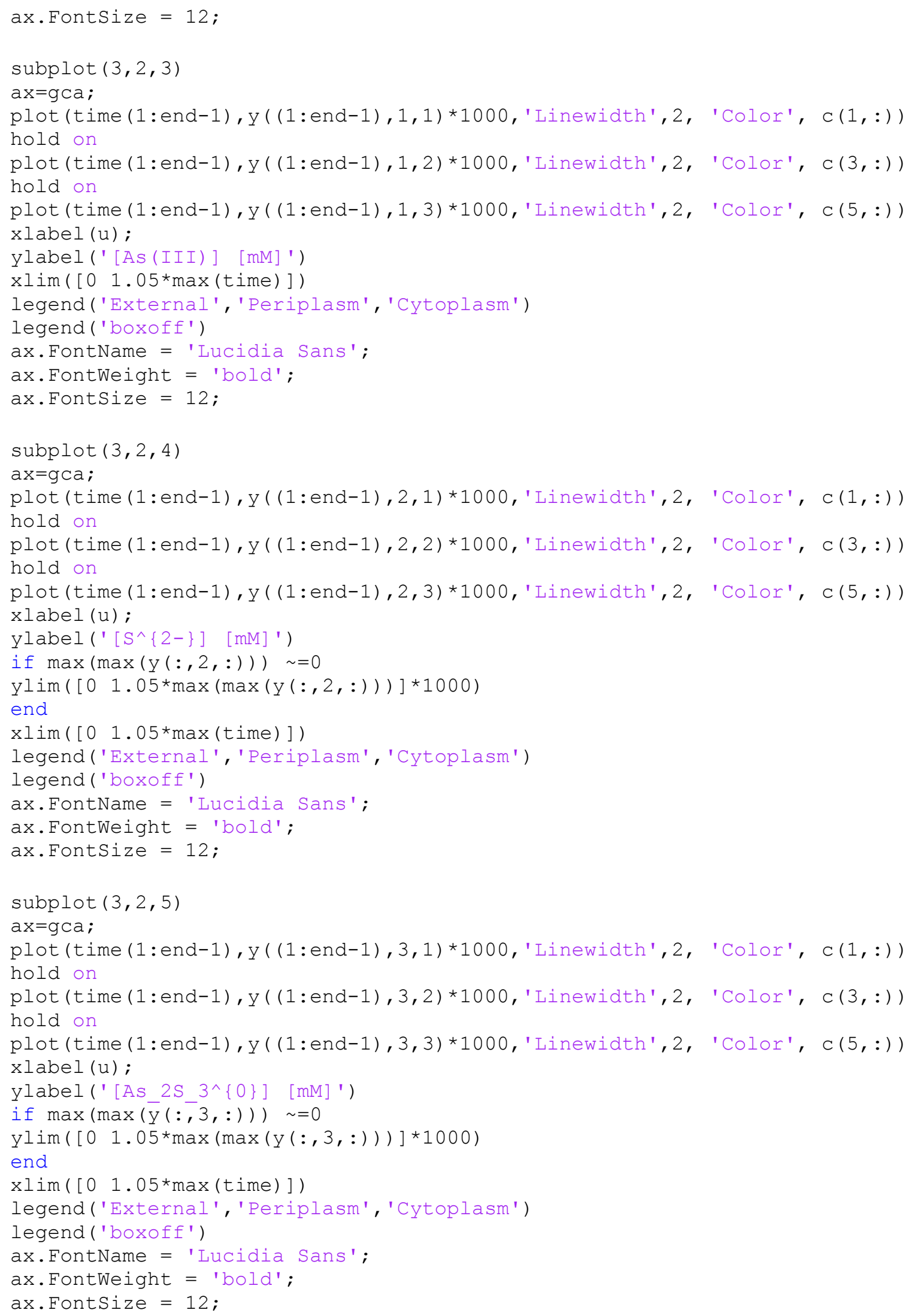




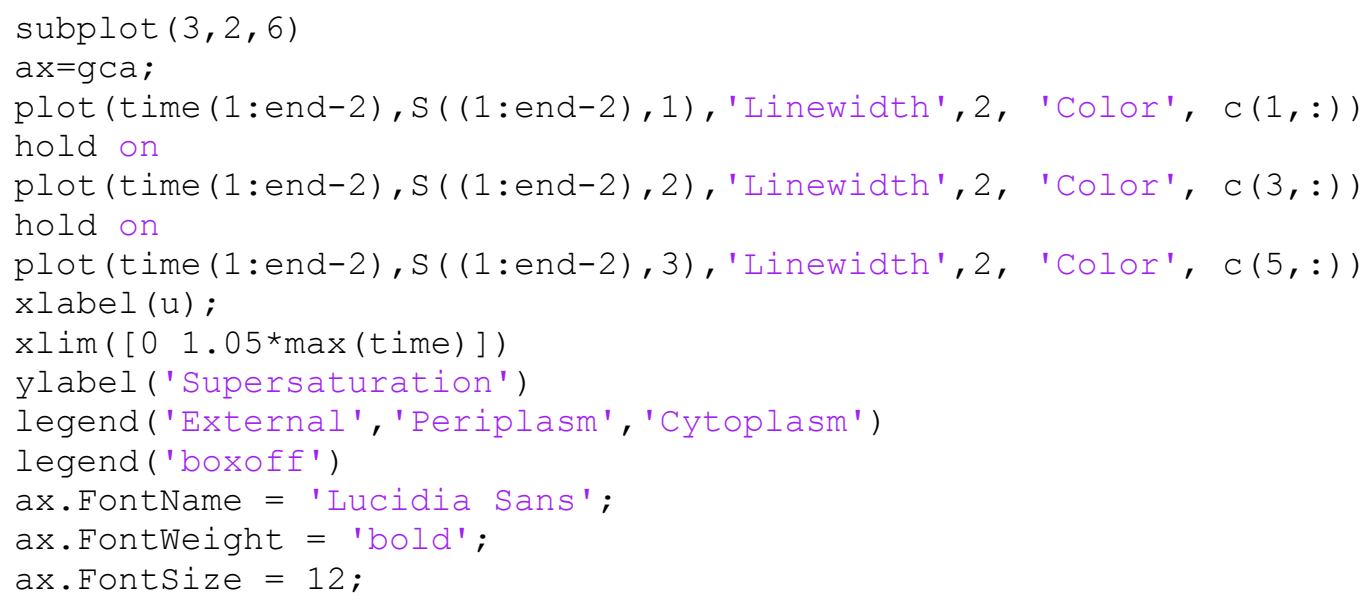

